\title{
The Correlation Analysis of Clean Energy Output Based on Nonparametric Kernel Density Estimation Probability Models
}

\author{
Xiaolin Qin ${ }^{1}$, Yonggang $\mathrm{Li}^{1}$, Chao Shen ${ }^{1}$, Zengqiang Zhang ${ }^{2}$ and Xuyao Zeng ${ }^{3}$ \\ ${ }^{1}$ School of Electrical and Electronic Engineering, North China Electric Power University, Baoding, China \\ ${ }^{2}$ Xinjiang Electric Power Company Economic Research Institute, Urumqi, China \\ ${ }^{3}$ Liujiaxia Hydropower Plant, Gansu Electric Power Company of State Grid Corporation of China, Yongjing, China
}

\begin{abstract}
There often exists correlation among adjacent photovoltaic power stations and wind farms. This paper provides a method based on the nonparametric kernel density estimation theory and Spearman rank correlation to analysis the correlation of clean energy outputs. Firstly, a variable bandwidth kernel density estimation method based on boundary kernel algorithm is built to establish the clean energy probability density model. The method can solve the boundary deviation and reflect the thick tail characteristics of clean energy, which improves the accuracy and practicability of the probability estimation. Next, some data from photovoltaic stations and wind farms of a certain power grid in Northwest China are used to obtain the probability density using above method, and simulated outputs of different wind farms and photovoltaic power stations. Then the complementary between clean energy's outputs are analyzed by using Spearman rank correlation coefficient. It can be concluded from the results that the probability models established by the nonparametric kernel density estimation theory can improve the accuracy and practicability of the probability estimations and the Spearman rank correlation coefficient can effectively reflect the output correlation of clean energy.
\end{abstract}

Keywords-Nonparametric kernel density estimation; variable bandwidth; clean energy; Spearman rank correlation coefficient

\section{INTRODUCTION}

Recent years, clean energy such as wind power and solar energy has become the important role in the field of electric energy, which will also in rapid development in the foreseeable future. However, the inherent characteristics of wind and solar power such as randomness and intermittent affect power system security and stability [1]. In addition, there are lack of corresponding overall planning to the actual power grid architecture when constructing clean energy stations, as a result, a high proportion of wind power and photovoltaic power is abandoned in the region where has large-scale clean energy. So, to research clean energy's random distribution and the correlation among clean energy will be of great significance in the planning and operation for power grid [2].

In terms of the randomness and intermittent characteristics of clean energy, experts at home and abroad launched a number of studies to modeling probability for clean energy output. At present, the probability density model is usually divided into two kinds: parametric estimation and nonparametric estimation.
Parametric estimation based on experience, that is to say, wind and solar radiation is assumed to be a known type parameter, then according to historical data to estimate the specific parameters and finally establish the output probability model of wind power and photovoltaic stations [3]. While, nonparametric estimation with no need for any prior assumption, it is a method driven by sample data to study the data distribution [4]. In the beginning period of the construction of clean energy, there is lack of actual output data, we can only indirectly rely on experience and artificial hypothesis to set up output probability model for clean energy. With the development of clean energy, we can easily collect minutes and seconds output data of wind power and photovoltaic stations, thus we can directly analyze the practical operation data in nonparametric estimation method to study the probability distribution of clean energy.

Nonparametric estimation method dates from histogram, and then derive many species: the nearest neighbor method, Rosenblatt method、kernel density estimation and so on [5]. Among them, the kernel density estimation is comparatively mature, which can effectively improve the accuracy of estimation and it is the main direction in the field of current nonparametric estimation method.

This paper will based on nonparametric kernel density estimation (KDE) theory to establish the probability models of clean energy output in the ground of the historical data in northwest China. And will put forward a variable bandwidth kernel density estimation based on boundary kernel algorithm to solve the problems of boundary bias and lacking of local adaptability.

In terms of correlation analysis, this paper use Spearman rank correlation [6] to calculate and analyze the complementary between clean energy's output over different time scale on the basis of above models and reach the regular of wind power and photovoltaic power stations, which is of great significance for clean energy planning of power grid in the future. 


\section{THE VARIABLE BANDWIDTH KeRNEL DENSITY ESTIMATION BASED ON BOUNDARY KERNEL ALGORITHM}

\section{A. The Method of Conventional KDE}

First, we put wind power as an example to deep exposition the principle of conventional nonparametric kernel density estimation:

$p_{1}, p_{2}, \ldots, p_{n}$ are $\mathrm{n}$ samples of wind power output, which is between $[\mathrm{a}, \mathrm{b}]$, and the nonparametric kernel density estimation probability of wind power can be written as (1)

$$
\hat{f(p)}=\frac{1}{n h^{d}} \sum_{i=1}^{n} K\left(\frac{p-p_{i}}{h}\right)
$$

where $f(p)$ is the estimate probability density, $h$ is the bandwidth of the estimation, $\mathrm{d}$ is the dimension of data space, and $K(p)$ is the kernel function of the estimation.

In order to ensure that $\hat{f(p)}$ is reasonable as the density function, $K(p)$ deed to satisfy:

- $K(p)$ must be smooth non-negative symmetric function

- $\quad \int K(p) d p=1$

- $\quad \int p K(p) d p=0$

- $\quad \int p^{2} K(p) d p=\sigma_{k}^{2}>0$

There are many kernel functions meet above conditions, and the conventional kernel functions are listed in table I.

TABLE I. THE CONVENTIONAL KERNEL FUNCTIONS

\begin{tabular}{cc}
\hline The Type of Kernel Function & Expression \\
\hline Uniform(Box) & $(1-|u|) I(|u| \leq 1)$ \\
Triangle & $\frac{3}{4}\left(1-u^{2}\right) I(|u| \leq 1)$ \\
Epanechnikov & $\frac{15}{16}\left(1-u^{2}\right)^{2} I(|u| \leq 1)$ \\
Quaritic & $\frac{35}{32}\left(1-u^{2}\right)^{3} I(|u| \leq 1)$ \\
Triweight & $\frac{1}{\sqrt{2 \pi}} \exp \left(-\frac{1}{2} u^{2}\right)$ \\
Gaussian & $\frac{\pi}{4} \cos \left(\frac{\pi}{2} u\right) I(|u| \leq 1)$ \\
Cosinus &
\end{tabular}

Literature [7] pointed out that different kernel functions have almost no effect on the result of nonparametric kernel density estimation.

Different from kernel functions, bandwidth has more effect on the accuracy of kernel density estimation: When $h$ is too large, it may lead to a highly smooth probability density and there are errors with actual values, while $h$ is too small, it can improve the precision of nonparametric kernel density estimation but will lead to a prohibitively volatility of kernel density estimation, therefore, the choice of the bandwidth must be balanced. Researchers usually adopt mean integrated squared error (MISE) to select the best bandwidth in practice, and it is calculated via cross-validation method, which is proposed by Rudemo (1982) and Bowman (1984).

$$
E_{\text {MISE }}\left(\hat{f}_{h}\right)=E\left\{\int[\hat{f(p)}-f(p)]^{2} d p\right\}
$$

When the sample size is big enough, namely $n h \rightarrow+\infty$, formula (5) can be written as:

$$
E_{\text {MISE }}\left(\hat{f}_{h}\right) \approx \frac{1}{4} \sigma_{k}{ }^{4} h^{4} \int\left[f^{\prime \prime}(p)\right]^{2} d p+\frac{1}{n h} \int[K(p)]^{2} d p
$$

Take the first partial derivatives of formula (6), it will get the best bandwidth that make MISE minimum:

$$
\hat{h}=\left\{\frac{\int\left[K(p)^{2} d p\right]}{\sigma_{k}^{4} \int\left[f^{\prime \prime}(p)\right]^{2} d p}\right\}^{\frac{1}{5}} n^{-\frac{1}{5}}
$$

Formula (7) contains the second derivative of $f(p)$, but $f(p)$ is unknown, so it is necessary to estimate $\int\left[f^{\prime \prime}(p)\right]^{2} d p$ to get the suitable $\hat{h}$.

In particular, when the overall distribution submits to $N\left(\mu, \sigma^{2}\right)$, and the kernel function is Gaussian kernel function, formula (7) can be simplified as:

$$
\hat{h}=\left(\frac{4}{3}\right)^{\frac{1}{5}} \sigma n^{-\frac{1}{5}} \approx 1.059 \sigma n^{-\frac{1}{5}}
$$

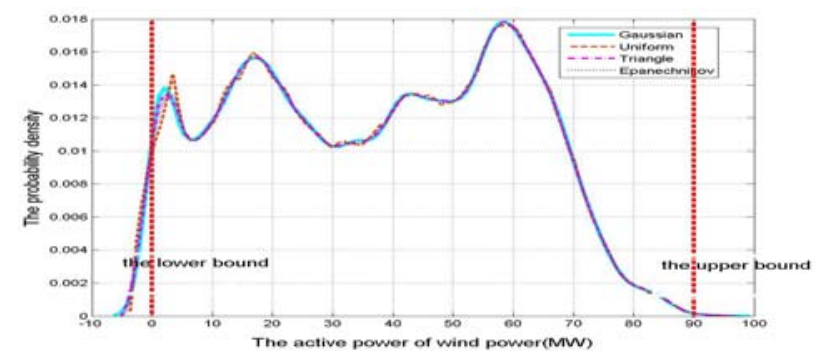




\section{FIGURE I. CONVENTIONAL KDE RESULTS UNDER DIFFERENT KERNEL FUNCTIONS ESTIMATION}

Figure I shows the results of kernel density estimation for a wind power (the capacity of which is $90 \mathrm{MW}$ ) output probability in four different kernel functions. It can be seen that four curves basically in coincidence, and in terms of smoothness, Gaussian kernel function and Epanechnikov kernel function are better than Triangle kernel function, but the Uniform kernel function is the worst. This paper will choose Gaussian kernel function as the kernel function of probability density estimation, combined with formula (1) and table I , the kernel density estimation of wind power's output probability can be written as:

$$
\hat{f(p)}=\frac{1}{\sqrt{2 \pi} n h} \sum_{i=1}^{n} \exp \left[-\frac{1}{2}\left(\frac{p-p_{i}}{h}\right)^{2}\right]
$$

Wind power's output have upper and lower bounds in actual operation, but it can be found from Figure I that conventional KDE algorithm exists deviation in the boundary (there is greater than 0 below 0MW as well as above 90MW ). In addition, the bandwidth of conventional $\mathrm{KDE}$ algorithm is fixed, and unable to reflect the characters of wind power in actual operation. Therefore, this paper put forward the following variable bandwidth kernel density estimation algorithm considering boundary kernel.

\section{B. The Improvement of Kernel Function}

In order to solve the problem of boundary deviation, this paper introduces "boundary kernel" function to modify the kernel function, whose expression is:

$$
B(p)=\frac{\left(q_{2}(p)-q_{1}(p)\right) K(p)}{q_{0}(p) q_{2}(p)-q_{1}^{2}(p)}
$$

where $q_{i}(p)=\int_{(x-b) / h}^{(x-a) / h} p^{i} K(p) d p$

It's okay to substitute $K(p)$ in formula (1) for $B(p)$ in formula (10), and the kernel density estimation algorithm considering boundary kernel as follows:

$$
\hat{f(p)}=\frac{1}{n h^{d}} \sum_{i=1}^{n} B\left(\frac{p-p_{i}}{h}\right)
$$

\section{The Slide Bandwidth Based on Estimate Point}

It can be seen that the value of $\hat{h}$ is closely related with $f(p)$ from formula (7), and the bandwidth changes follow the value of $p$, but the optimization of bandwidth in above ways is the result over the entire range, which will reduce the accuracy of probability density estimation obviously. This paper substitutes $f(p)$ for $\hat{f(p)}$ considering that $f(p)$ is unknown, and then get the value of $\hat{h}$ of each point via formula (7). For example, when the kernel function is Gaussian kernel:

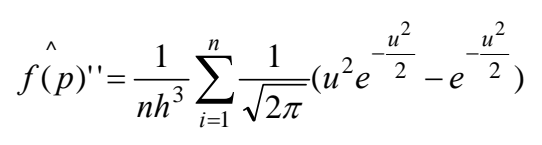

where $u=\frac{p-p_{i}}{h}$, and $h$ is the fixed bandwidth through the conventional method.

Combined with formula (7):

$$
h(p)=\frac{h^{1.8} \int f^{\prime \prime}(p) d p^{0.2} \hat{f(p)}^{0.2}}{\left[\sum_{i=1}^{n} \frac{1}{n h} u^{2} K(p)-\hat{f(p)]^{0.4}}\right.}
$$

So we eventually get the variable bandwidth kernel density estimation based on boundary kernel algorithm:

$$
\hat{f(p)}=\frac{1}{n h(p)^{d}} \sum_{i=1}^{n} B\left(\frac{p-p_{i}}{h(p)}\right)
$$

\section{THE CORRELATION COEFFICIENT AND ITS MATRIX OF ClEAN ENERGy OUTPUT}

The so called Pearson coefficient was the first formal correlation measure, a measure of a linear relationship between the variables, is still one of the most broadly applied indices in statistics. But we learn that the wind farms and photovoltaic power stations output are not conform to the linear assumption, so we use another measure of the degree of association between two variables, namely the Spearman rank correlation coefficient. When the data are in the form of ranks, to measure the degree of association between the two variables, the so called the Spearman rank correlation coefficient is used [8].

Let $\left(R_{1}, S_{1}\right),\left(R_{2}, S_{2}\right), \ldots,\left(R_{n}, S_{n}\right)$ be a random sample of size n. To compute the Spearman correlation coefficient we rank all the observations of the first variable. Next, we independently rank the values of the second variable. The Spearman rank correlation coefficient is the Pearson correlation coefficient applied to the ranks R, namely:

$$
q_{r s}=\frac{\sum_{i=1}^{n}\left(R_{i}-\bar{R}\right)\left(S_{i}-\bar{S}\right)}{\sqrt{\sum_{i=1}^{n}\left(R_{i}-\bar{R}\right)^{2}} \sqrt{\sum_{i=1}^{n}\left(S_{i}-\bar{S}\right)^{2}}}
$$

where $\bar{R}=\frac{1}{n} \sum_{i=1}^{n} R_{i}, \bar{S}=\frac{1}{n} \sum_{i=1}^{n} S_{i}$. 
When there are not two values of $\mathrm{R}$ or two values of $\mathrm{S}$ with the same rank (so called ties), there is an easier way to compute the Spearman correlation coefficient:

$$
q_{r s}=1-\frac{6}{n\left(n^{2}-1\right)} \sum_{i=1}^{n} d_{i}^{2}
$$

where $d_{i}=R_{i}-S_{i}, \quad i=1,2, \ldots, n$.

If there are two values of $\mathrm{R}$ or two values of $\mathrm{S}$ with the same rank, but the number is small compared with $n$, (17) is still useful, and $-1 \leq q_{r s} \leq 1$.

The relationship between the value of Spearman and the degree of association is shown in table II.

\begin{tabular}{ccc}
$\begin{array}{c}\text { TABLE II. THE RELATIONSHIP BETWEEN THE VALUE OF } \\
\text { SPEARMAN AND THE DEGREE OF ASSOCIATION }\end{array}$ & $\begin{array}{c}\text { Negative value } \\
\text { (complementarit } \\
\text { The Degree of } \\
\text { association }\end{array}$ & $\begin{array}{c}\text { Positive value } \\
\text { (consistency) }\end{array}$ \\
\hline $\begin{array}{c}\text { No relation } \\
\text { Low correlation }\end{array}$ & 0 & 0 \\
$\begin{array}{c}\text { Moderate correlation } \\
\text { High correlation } \\
\text { Completely } \\
\text { correlation }\end{array}$ & $(-0.3-0)$ & $(0-0.3]$ \\
$(-1.0-0.3-0.7]$ & {$[0.7-1.0)$} \\
\end{tabular}

When research the correlation relationship between clean energies over a wide area, we will involve different wind farms in the same wind area and different wind areas. In order to clearly describe the interactions between multivariate stochastic variables, we introduce the matrix of Spearman rank correlation coefficient:

$$
\mathrm{P}=\left[\begin{array}{cccc}
1 & \rho_{12} & \cdots & \rho_{1 M} \\
\rho_{21} & 1 & \cdots & \rho_{2 M} \\
\vdots & \vdots & \ddots & \vdots \\
\rho_{M 1} & \rho_{M 2} & \cdots & 1
\end{array}\right]
$$

where $\rho_{i j}$ is the Spearman rank correlation coefficient between $\mathrm{R}_{\mathrm{i}}$ and $\mathrm{S}_{\mathrm{j}}$.

\section{EXAMPLE ANALYSIS}

This paper uses the statistical data of grid power in Northwest China (June 2015 to August 2015) to establish the variable bandwidth kernel density estimation model of wind power and photovoltaic power, based which we can calculate the Spearman rank correlation coefficient among different wind power farms and photovoltaic power stations.
A. Analysis for the Probability Density Model Results of Clean Energy

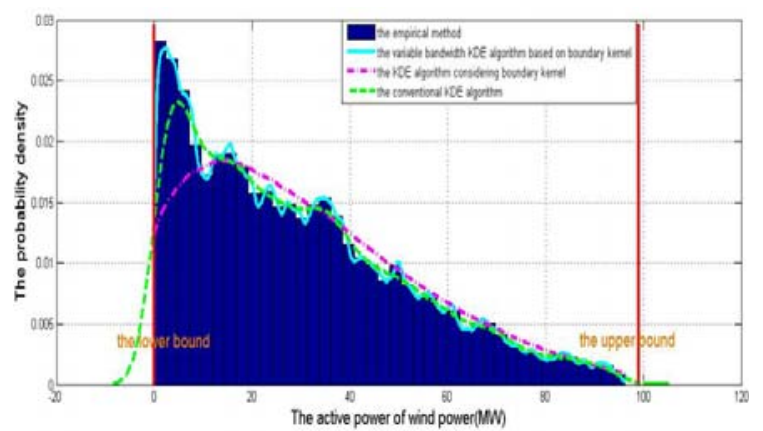

FIGURE II. THE PROBABILITY DENSITY ESTIMATION RESULTS

Figure II is the curve of the probability density for a wind power farm whose capacity is $99 \mathrm{MW}$. And there are results of empirical method、conventional KDE algorithm as well as the method proposed by this paper, we can draw the following conclusions:

- The algorithm considering boundary kernel solves the problem of boundary deviation exists in conventional $\mathrm{KDE}$ algorithm, and reflects the actual situation.

- Compared with the fixed bandwidth algorithm, the variable bandwidth algorithm is more match with the results of empirical method, we can see it from Figure II that the bandwidth slide based on estimate point in the method proposed by this paper, and solve the problem that lacking local adaptability in fixed bandwidth algorithm.

B. Analysis of Correlation Among Different Wind Farms in the Same Wind Area

First, we select four different wind farms' output in the same wind area and raise the kernel density estimation probability model, then we simulate daily output and monthly output for wind farms, finally we calculate the Spearman rank correlation coefficient matrix as follows:

$$
\left[\begin{array}{cccc}
1 & 0.436 & 0.461 & 0.559 \\
0.436 & 1 & 0.890 & 0.815 \\
0.461 & 0.890 & 1 & 0.847 \\
0.559 & 0.815 & 0.847 & 1
\end{array}\right]
$$

(a) The correlation coefficient matrix of daily output

$$
\left[\begin{array}{cccc}
1 & 0.627 & 0.713 & 0.679 \\
0.627 & 1 & 0.901 & 0.848 \\
0.713 & 0.901 & 1 & 0.896 \\
0.679 & 0.848 & 0.896 & 1
\end{array}\right]
$$

(b) The correlation coefficient matrix of monthly output 
It can be found that different wind farms' output in the same wind area has stronger positive correlation, and it is more apparent in monthly output than daily output. Therefore, when we study the laws of fluctuations of wind farms in the specific wind area under the background of large-scale grid wind power, it can be seen as a group with highly consistent correlation coefficient and have no need to consider the effect for the system of each wind farm.

\section{Analysis of Correlation Among Different Wind Farms in Different Wind Areas}

We simulate daily output and monthly output for the typical wind farm of wind area 1 to 7 , and the Spearman rank correlation coefficient matrix is calculated as follows:

$\left[\begin{array}{ccccccc}1 & -0.008 & 0.077 & 0.023 & 0.372 & 0.117 & 0.264 \\ -0.008 & 1 & 0.097 & 0.054 & 0.003 & -0.023 & 0.119 \\ 0.077 & 0.097 & 1 & 0.19 & 0.055 & 0.13 & 0.196 \\ 0.023 & 0.054 & 0.19 & 1 & -0.147 & -0.019 & -0.092 \\ 0.372 & 0.003 & 0.055 & -0.147 & 1 & 0.253 & 0.256 \\ 0.117 & -0.023 & 0.13 & -0.019 & 0.253 & 1 & 0.119 \\ 0.264 & 0.119 & 0.196 & -0.092 & 0.256 & 0.119 & 1\end{array}\right]$

(a) The correlation coefficient matrix of daily output

$$
\left[\begin{array}{ccccccc}
1 & 0.13 & 0.06 & 0.203 & -0.083 & 0.128 & 0.387 \\
0.13 & 1 & -0.154 & 0.223 & 0.305 & 0.182 & -0.048 \\
0.06 & -0.154 & 1 & 0.008 & 0.073 & -0.072 & 0.06 \\
0.203 & 0.223 & 0.008 & 1 & 0.127 & 0.256 & -0.019 \\
-0.083 & 0.305 & 0.073 & 0.127 & 1 & 0.089 & -0.014 \\
0.128 & 0.182 & -0.072 & 0.256 & 0.089 & 1 & -0.112 \\
0.387 & -0.048 & 0.06 & -0.019 & -0.014 & -0.112 & 1
\end{array}\right]
$$

(b) The correlation coefficient matrix of monthly output

Compared with the result of different wind farms' output in the same wind area, there is a obviously decrease of the Spearman rank correlation coefficient among different wind farms in different wind areas (whose maximum is 0.387), and there are negative values in the matrix, which indicate that the wind farms output exist much differences in different wind areas, and they are complementary when they joint operation.

\section{Analysis of Correlation Among Different Photovoltaic Power Stations in Different Areas}

Photovoltaic powers' output is sensitive to the light intensity, and we simulate monthly output for the typical photovoltaic power stations output from west to east, and the Spearman rank correlation coefficient matrix is calculated as follows:
Obviously, it has stronger positive correlation of photovoltaic powers' output, and further analysis can be concluded that the value of correlation coefficient decrease with the distance increase, which is more regularly than the wind power.

\section{CONCLUSION}

Based on the nonparametric kernel density estimation theory, this paper established the variable bandwidth kernel density estimation model considering boundary kernel algorithm, can solve the problems of boundary deviation and lacking of local adaptability in Conventional KDE algorithm, and chose the actual output of wind farms and in Northwest China as an example to build the kernel density estimation model, the simulation result demonstrates the effectiveness and adaptability of the proposed method.

Simulated different wind farms and photovoltaic power stations output according to the model proposed in this paper, and the correlations were calculated through Spearman rank correlation coefficient of clean energies in large-scale grid, and summarized the correlation rule among different wind farms in the same wind area and different wind areas as well as different photovoltaic power stations in different areas, from which we can lay the foundation for the output characteristics of largescale clean energy.

\section{REFERENCES}

[1] Jun Zhang, Xiaoyan Xu, Yongning Huang, Yang Cao and Yujia Gu, "Optimal proportion study of wind and PV capacity in Ningxia power grid based on time sequence simulation,” in Power System Protection and Control , vol. 42, pp. 81-86, 2014.

[2] Yuqin $\mathrm{Xu}$, Kun Chen, Junqing $\mathrm{Li}$ and Yang Nie, "A New Method Analyzing Output Correlation of Multi-Wind Farms Based on Combination of Copula Function and Kernel Estimation Theory,"[J]. in Transactions of China Electrotechnical Society, vol. 31 pp. 92-100 2016.

[3] Yuanzhang Sun, Jun Wu, Guojie Li, et al. "Dynamic economic dispatch considering wind power penetration based on wind speed forecasting and stochastic programming," in Proceedings of the CSEE, vol. 29, pp. 41-47,2009.

[4] Rosenblatt M, "Remarks on some nonparametric estimates of a density function,” Annals of Mathematical Statistics, vol. 31,pp.832-837,1956.

[5] Parzen E, "On estimation of a probability density function and mode," Annals of Mathematical Statistics, vol. 33, pp. 1065-1076, 1962.

[6] Genest C. Maekay J, "The joy of copulas:Bivariate distributions with uniform marginals," in The American Statistician, vol. 40, pp.280283,1986.

[7] Epanechnikov V A, "Nonparametric estimation of a multidimensional porability density," in The Theory of Porability and Application, 1969 vol. 14 , pp.153-158, 1969.

[8] Rodgers J. L. and W. Alan Nicewander W. A. "Thirteen Ways to Look at the Correlation Coefficient,” in The American Statistician, vol. 42, pp. 59-66, 1988.

$$
\left[\begin{array}{cccc}
1 & 0.972 & 0.936 & 0.917 \\
0.972 & 1 & 0.941 & 0.92 \\
0.936 & 0.941 & 1 & 0.99 \\
0.917 & 0.92 & 0.99 & 1
\end{array}\right]
$$

